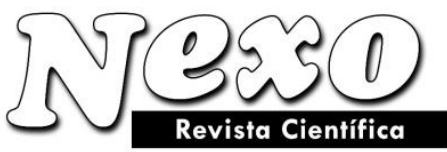

ISSN-E 1995-9516

Universidad Nacional de Ingeniería COPYRIGHT @ (UNI). TODOS LOS DERECHOS RESERVADOS http://revistas.uni.edu.ni/index.php/Nexo https://doi.org/10.5377/nexo.v34i05.13111

Vol. 34, No. 05 (Especial), pp. 75-82/Diciembre 2021

\title{
¿Arquitectura, parte integral de las Ciencias sociales?
}

\section{¿Architecture, an integral part of Social sciences?}

\author{
Fabian E. Báez Álvarez ${ }^{1, *}$, Katherine del C. González Rivera ${ }^{2}$ \\ ${ }^{1}$ Universidad La Gran Colombia. Facultad de Arquitectura, Programa de Arquitectura. Bogotá, Colombia. \\ *abian.baez@ugc.edu.co \\ ${ }^{2}$ Universidad Autónoma de Chiriquí, Facultad de Arquitectura. Licenciatura en Arquitectura. David, \\ Panamá. \\ katherine.gonzalez8@unachi.ac.pa
}

(recibido/received: 13-septiembre-2021; aceptado/accepted: 23-noviembre-2021)

\section{RESUMEN}

La identificación de la arquitectura como profesión siempre ha generado dicotomías educacionales ya que se ha considerado esta profesión como parte de las bellas artes, sin embargo, su carácter organizador del espacio, hace que ella se concentre en esa labor edificatoria y/o constructiva, volcándola hacia otra clasificación dentro del campo de la educación donde se incorpora como ingeniería, industria, tecnología y construcción; no obstante, se identifica siempre una intervención del "ser para el ser", ubicando a esta no solamente dentro del contexto espacial, sino del confort ambiental y social, pues es en este último donde se mueve la labor del arquitecto, donde muchas veces se requiere de esta ciencia para analizar, identificar, caracterizar e incluso cuantificar al usuario, desde su comportamiento humano como individuo y como grupo. Es por ello, que de allí nace el título de este artículo en forma de pregunta: ¿Arquitectura, parte integral de las ciencias sociales?, este tema se planteara de forma crítica y dialéctica, generando un excelente acercamiento y comprendiendo aún mejor a esta gran profesión.

Palabras claves: Arquitectura, teoría de la arquitectura, ciencias sociales, educación, lenguaje arquitectónico.

\section{ABSTRACT}

The identification of architecture as a profession has always generated educational dichotomies since this profession has been considered as part of the fine arts, however, its organizing nature of space, makes it concentrate on that edifying and / or constructive work, overturning it towards another classification within the field of education where it is incorporated as engineering, industry, technology and construction; However, an intervention of "being for being" is always identified, placing it not only within the spatial context, but also within the environmental and social comfort, since it is in the latter where the architect's work moves, where many times it is it requires this science to analyze, identify, characterize and even quantify the user, from his human behavior as an individual and as a group. That is why the title of this article was born from there in the form of a question: Architecture, an integral part of the social sciences? This topic will be raised critically and dialectically, generating an excellent approach and understanding this great profession.

Keywords: Architecture, architecture theory, social sciences, education, architectural language. 


\section{INTRODUCCIÓN}

Como parte de la labor docente compete no solamente el de transmitir conocimientos a los futuros colegas, sino formar parte del discernir, confrontar e incluso contradecir, algunos criterios que desde la escuela se ven como grandes verdades, pero que en realidad son magnos paradigmas que requieren de un análisis o de una interpretación ya que por sí solos se sostienen, y esto es debido a una falta de crítica que reinvencione su valor, es por ello que, a través de estas líneas, se quiere realizar una identificación del que es ser arquitecto, comprender aún mas que es la arquitectura, como profesión y su consecuencia social sobre el ser humano.

Para ello, se ha propuesto una revisión documental que para efectos de lectura su trazabilidad es cronológica, que presentara la incidencia del objeto diseñado por parte del arquitecto, sobre el sujeto o sujetos que lo usarán, y cómo afecta a estos desde su realidad alrededor de su rol participante de un medio físico y social creado, debido a unas determinantes impuestas por unos estereotipos normativos y de tendencias de moda.

De allí la pregunta título: ¿Arquitectura, parte integral de las ciencias sociales?, cuestión que nos atañe el de reconocer e identificar, más allá de una clasificación realizada por la Organización de las Naciones Unidas para la Educación, la Ciencia y la Cultura - UNESCO, donde se clasifica la arquitectura dentro de otra área del conocimiento que incluso es más joven que ella, relegando a esta profesión a una subordinación impuesta que podría, contradecir su valor inicial vital, correspondiente a la integración del diseño arquitectónico al bienestar social de las comunidades.

Se agota esta primera parte introductoria con el siguiente fragmento, donde también se habla de arquitectura:

Luis Piette, arqueólogo francés, ha ordenado en la serie que lleva su nombre las actividades artísticas de la prehistoria. Se advierte en ella cómo halló el hombre, en su propio cuerpo, campo propicio para sus afanes de arte. Cicatrices y tatuajes diéronle así una suerte de personalidad física en las sociedades primitivas, cuyos artistas crearon después y sucesivamente: La escultura de bulto y el bajo relieve, el dibujo y la pintura la cerámica, copiando formas humanas y luego formas animales, y, finalmente, la composición ornamental geométrica. Bajo el signo formal de la geometría habría de surgir también, pero posteriormente, la Arquitectura, cerrándose así el cuadro de las artes plásticas y demostrándose, a la par, que la actividad inteligente del hombre señoreó, antes que otros campos, los de su sensibilidad ante la belleza, fundamento del arte (Jackson, 1952, p.65).

\section{ARQUITECTURA MÁS ALLA DE LA ESPACIALIDAD.}

Como parte de la labor documental se extractaron cuatro conceptos sobre arquitectura inscritos a través del tiempo, buscando de manera reflexiva una filiación profesional en pasado, presente y posiblemente futura, inscribiéndola dentro de una dinámica de acción del ejercicio y quehacer profesional, se busca con ello, realizar una identidad de discernimientos, que nutran conceptos y visiones más allá de la espacialidad.

Se inicia este recorrido a través del arquitecto británico creador del arts and crafts, William Morris:

La arquitectura abarca la consideración de todo el ambiente físico que rodea la vida humana; no podemos sustraernos a ella, mientras formemos parte de la civilización, porque la arquitectura es el conjunto de modificaciones y alteraciones introducidas en la superficie terrestre con objeto de satisfacer las necesidades humanas, exceptuando solo al puro desierto (Benévolo, 1982, p.6).

Se reconoce al arquitecto William Morris como un visionario en la economía, ya que concibe el placer del trabajo como una labor llena de sensibilidad y habilidad, de allí la creación de la Escuela de Artes y Oficios, como parte del equilibrio en contraposición con la producción en masa presentada en ese momento por el 
auge de la Revolución Industrial, donde nunca prima la calidad del producto, ni el costo y la exquisitez por la originalidad; de otra parte influye en la naturaleza del consumidor, ya que considera que este debe obtener una educación comercial, incentivando al individuo en que reconozca lo que necesita y se apropie del producto; por lo anterior, vemos como se vincula la temática económica con una interacción sobre la ciudad como espacio donde se ejecutan todas estas relaciones comerciales, de plano se habla de necesidades humanas, definida por Manfred Max Neef en dos tipos de categorías, la primera de estas corresponde a las necesidades del ser, tener, hacer y estar; y como segunda categoría, las necesidades de subsistencia, protección, afecto, entendimiento, participación, ocio, creación, identidad y libertad; principios fundamentales para un verdadero equilibrio del ser humano. En lo único en que desacierta Morris en su definición es la exclusión del desierto como parte de la incorporación urbana, no obstante, se entiende su punto de vista ya que en el siglo XVIII este espacio era considerado sin vida y peor aún de una vida urbana.

Como segundo axioma se presenta en términos enciclopédicos, esta es la interpretación de ese momento:

Arquitectura, procedente del término del griego arches (principal) y tecton (obra) sufijo -ure (actividad o resultado), que significa preeminencia sobre quienes realizan técnicas constructivas que los obreros construyen; el arquitecto por su parte es hoy un profesional, cuyo título recibe, según los países, de las universidades o de las escuelas especiales; y cuya actividad se ejerce, atentas modalidades diferentes, con carácter de libre o sujeta a reglamentación. Confundida muy frecuentemente con la del ingeniero (calificado usualmente como civil) y con la de los maestros de obras (denominado en léxico popular como constructor), la misión del arquitecto ni es de técnica pura ni constituye una actividad industrial. Su habilidad para componer en el proyecto y para dirigir en la ejecución representa una manera peculiar de actividad, interpolada entre las otras dos; y que, aspirando a ser artística, se funda en la técnica necesaria, hoy procedente de la ingeniería, y en la previsión económica suficiente para erigir un edificio (Jackson, 1952, p.67).

Del axioma anterior, se podría identificar la arquitectura y al arquitecto, como esa realidad de producto edificatorio con cierta formalidad plástica, circunscribiéndola en un mero objeto de utilidad funcional en beneficio para una habitabilidad permanente o pasajera, sin que se analicen las características y/o necesidades del usuario; se resalta la estabilidad de la obra arquitectónica a través de una buena ingeniería, convirtiéndose esta profesión en un agente diseñador y ejecutor, sin un episodio de la identificación de la incidencia del objeto construido sobre su entorno inmediato, ni mucho menos de un estudio de impacto sobre la sociedad a la que interviene.

Es por ello, que se tiende a confundir entre el ejercicio del ingeniero civil y el del maestro de obra, los cuales son bien apreciados en la labor edificatoria del objeto arquitectónico, cada uno con sus propias cualidades experticias que podríamos resumir el primero en su gran labor estructural y el segundo como ejecutor tanto de la estructura como del envolvente de la obra unido al desarrollo de los detalles que enriquecen la edificación; la presencia del arquitecto se fundamenta en su gran visión por engalanar su obra como director del ejercicio ya que, en él recae la perfecta coherencia entre la construcción y su entorno inmediato; de este axioma podríamos decir que se nota la frialdad por solo convertir la arquitectura en la modestia de un edificio sin que intervenga ningún valor social.

El hecho que se elude hoy el problema de un lenguaje propio de la arquitectura demuestra que quizá por el momento hemos sido capaces de deshacernos de él, pero esto no quiere decir que tal cuestión no se plantee hoy en los mismos e idénticos términos de entonces. Por su parte, como lo explica Grassi:

Más aún: los arquitectos del pasado, los tratadistas, por ejemplo, hablan de la arquitectura como algo claramente delimitado y definido que va a añadirse a un patrimonio existente. Hablan de la arquitectura con la familiaridad y la regularidad con la que cada hombre habla de su propio trabajo. No existe separación delimitada entre el lenguaje que usan las obras, de tal manera que los textos 
tienen una claridad igual a la evidencia de sus edificios. El lenguaje usado es parte inseparable de los elementos mismos del oficio, y por ello relativo a los principios, a las técnicas, a los materiales y al modo de usarlos, a los elementos de la arquitectura y a las relaciones que entre éstos se han establecido en las obras (como se cita en Patteta (1997, p.76).

La incorporación de la palabra lenguaje demuestra una arquitectura con gran capacidad de expresión de pensamientos y sentimientos, a través de ese creador diseñador, expresada en una materialidad interpretadora de la comunicación entre sociedad, espacio y objeto construido podríamos decir que este lenguaje se irradia en nueve tipos de lenguaje a saber: Lenguaje Conceptual, Lenguaje Semiótico, Lenguaje Simbólico, Lenguaje Formal, Lenguaje Funcional, Lenguaje Espacial, Lenguaje Contextual, Lenguaje Constructivo y Lenguaje Tecnológico Ambiental. Que se proponen a continuación:

- Lenguaje Conceptual: Se considera este lenguaje como la primera fase del diseño arquitectónico, ya que en el influye el primer concepto que tiene el arquitecto en su mente del objeto a transformar con sus respectivas cualidades, teniendo en cuenta el sitio de implantación.

- Lenguaje Semiótico: Los signos de comunicación en el desarrollo del diseño arquitectónico se ve evidenciado en efectos que sugiere el diseñador a través de la circulación y el uso de barreras físicas o creadas, para inducir al usuario a reconocer la espacialidad sus límites y propiedades.

- Lenguaje Simbólico: El objeto arquitectónico se convierte en una representación o hito dentro de la ciudad, por lo tanto, he allí el gran valor de este lenguaje, muchas veces no requiere de ser esbelto, sino un buen uso de la materialidad e incluso de la forma para que cree ese impacto en el contexto.

- Lenguaje Formal: Las características que determina la planta arquitectónica muchas veces son el efecto de los tres lenguajes descritos anteriormente, desarrollados en ese cruce de líneas y formas que convierten ese elemento bidimensional en algo aún más elaborado, posteriormente pasará esa imagen a una realidad tridimensional, donde se tendrá en cuenta al usuario y su efecto de asombro ante el objeto arquitectónico.

- Lenguaje Funcional: La forma y la función deben estar correlacionadas, pues posiblemente existirán efectos donde la realidad de la forma no influye en la función, sino es la búsqueda por parte del diseñador en crear ambientes de confort e incluso de integración entre el interior y el exterior.

- Lenguaje Espacial: Se plantea como ese medio físico en el cual se implanta el hecho arquitectónico, correspondiente a ese tipo de terreno con características topográficas e incidencias del lugar junto con ese valor social que lo enriquece, a este lenguaje se le debe sumar siempre el tiempo como cuarta dimensión, pues trascienden acontecimientos que influyen en la vida del edificio.

- Lenguaje Contextual: Este lo conforman la interpretación histórica y social del objeto arquitectónico y su influencia sobre la escala urbana a nivel de manzana, barrial, vecinal, zonal, urbano o metropolitano.

- Lenguaje Constructivo: Es la materialización del hecho arquitectónico usando las diferentes técnicas constructivas, contrastando los diferentes materiales que dinamizaran entre el color y la textura de la obra, es en sí el punto clave y final donde redundan todos los lenguajes anteriormente descritos.

- Lenguaje Tecnológico Ambiental: Forma parte del compromiso con “nuestra casa común", la de usar todas las herramientas que estén a nuestro alcance para reducir la huella de carbono que pueda presentar la edificación.

Es sin lugar a duda que la vinculación de estos nueve lenguajes hace que la arquitectura tenga una mejor y llamativa connotación, pues expresa una razón de ser que trasciende más allá de un objeto creado, pues estos 
lenguajes tienen en cuenta múltiples facetas esenciales para la supervivencia de la edificación ante la ciudad y la sociedad.

Por último, se extrae la siguiente enunciación de lo que se entiende por arquitectura en el ámbito jurídico:

Para todos los efectos legales, entiéndase por arquitectura, la profesión a nivel universitario, cuya formación consiste en el arte de diseñar y crear espacios, de construir obras materiales para el uso y comodidad de los seres humanos, cuyo campo de acción se desarrolla fundamentalmente con un conjunto de principios técnicos y artísticos que regulan dicho arte (L. 435, art.1, 1998).

Dentro de esta definición se resalta la palabra "arte", de la cual queda más que demostrado que en términos conceptuales la arquitectura no redunda en esa especialidad, por el contrario, busca mayores dinámicas urbanas y constructivas en pro del beneficio particular y común del usuario permanente o transeúnte, de los espacios entorno a la edificación denominados espacio público o espacios privados correspondientes al objeto u hecho arquitectónico, por lo tanto, es la definición menos acertada y se direcciona únicamente hacia actividades demasiado técnicas sin incidencia al valor social pues esta se limita a la "comodidad humana" y no a las necesidades humanas como se ha expresado anteriormente.

Además se expresa de principios artísticos, muy diferentes a los principios de composición que realmente usa el arquitecto a nivel del lenguaje conceptual, combinados con el lenguaje semiótico y simbólico el cual se evidencia al momento del trazado del lenguaje formal de lo cual es lo único en que se acierta pues se habla de "diseñar y crear espacios", los otros lenguajes se ignoran, posiblemente por falta de actualización del contexto actual de la arquitectura, ya que esta carrera está evolucionando hacia una integralidad más amena con el usuario del siglo XXI, reconociéndolo como un agente cambiante social donde se deben transformar sus necesidades humanas, con el apoyo de las ciencias sociales como base fundamental de esta reinterpretación.

\section{USO DE LAS CIENCIAS SOCIALES EN ARQUITECTURA}

Anteriormente se realizó un recorrido para entender que es la arquitectura y que es ser un arquitecto, por otra parte, en este espacio se realizará un recorrido por definir que son las ciencias sociales y como ellas generan gran influencia dentro del desarrollo de la arquitectura tanto en su etapa investigativa como al momento de ejecutar el hecho arquitectónico, para ello nos apoyaremos de algunas citas del arquitecto Josep Montoñola Thorbeng, de su texto cuyo título es Psicología y Arquitectura. Notas Breves.

El identificar las Ciencias Sociales, como la expresión del conjunto de disciplinas que estudian los fenómenos relacionados con la situación del ser humano; se puede contar entre ellas la economía, la sociología, la antropología, la psicología, la historia, la geografía, entre otras. De esta amplitud de disciplinas es en la que el arquitecto debe basarse para el uso racional de la arquitectura y esto lo denota Montañola en la siguiente cita:

Las ciencias sociales podían ayudar a los arquitectos a conocerse a sí mismos y a plantearse los límites y a las posibilidades disciplinares de su propio trabajo, pero para ello habían de tomar distancia critica con respecto a sí mismos. En este punto las ciencias sociales podrían ser más útiles y hasta "practicas". Además, ello hubiera ayudado a que la profesión del arquitecto fuera más "comunicable" y más "sensible" con respecto a la sociedad en general, siempre que se mantuviera un significado compartido de las nociones y no de una "jerga" insignificante, bien desde el exterior, bien desde el interior de la profesión (1986, p.34).

Las Ciencias Sociales, son bien usadas dentro del diario vivir arquitectónico, iniciando con la geografía ya que esta nos ayuda tanto a ubicar como a localizar el proyecto arquitectónico, la historia nos ayuda a 
comprender la cronología el lugar y la incidencia del objeto arquitectónico en el lugar, las demás disciplinas relacionadas aportan cada una de ellas desde su campo convirtiéndose en valiosas al momento de entender al ser humano y su realidad. Del texto anterior se resalta la palabra "jerga", puesto que muchas veces dentro del acervo textual ingresamos muchas palabras sin contemplarse el verdadero significado, solamente las incorporamos porque son de uso habitual o posiblemente están de moda, realidades que se han visto de estas palabras con gran significado dentro de las Ciencias Sociales, que son mal citadas por los arquitectos que requieren de una mayor profundidad de estudio para que verdaderamente sean complemento dentro de la realidad social del hecho arquitectónico proyectado; bien lo menciona Montañola, la interpretación del uso de las Ciencias Sociales no solamente son extrínseco sino intrínseca tanto para la obra como para el arquitecto, ya que el arquitecto se debe conocer en cada realidad proyectual.

Por ello, es que los arquitectos son considerados vanguardistas, pues siempre están un paso adelante sobre tendencias ideológicas, políticas, económicas, literarias, artísticas, entre otras; pues estas influyen en sus creaciones, no obstante, pueden caer en la manipulación del uso de tendencias caprichosas fuera de contextos o incluso en sobre usar materiales no benéficos para sus creaciones arquitectónicas. Al respecto menciona Montañola:

Lo cierto es que en los tratados y libros de arquitectura los arquitectos han intentado siempre estar al día en sus conocimientos científicos, y estos conocimientos se han incorporado inmediatamente, a lo largo del siglo XVII, XVIII y XIX, lo más relevante de la naciente psicología (1986, p.35)

Para precisar que nos hace falta en este momento, se comparte la Tabla 1, denominada: Tendencias arquitectónicas modernas y piscología, que nos ayuda a visualizar que es lo que posiblemente está sucediendo en estos momentos y por qué un posible desapego por las Ciencias Sociales.

Tabla 1. Tendencias arquitectónicas modernas y psicológicas.

\begin{tabular}{cc}
\hline Arquitectura & Psicología \\
\hline Las tendencias expresionistas, & Influencia del psicoanálisis en sus \\
dadaístas y surrealistas. & diferentes formas. \\
Las tendencias del International Style & Psicología de la Gestalt y fisiologías de \\
(Bauhaus y Mies Van Der Rohe). & la percepción. \\
En tendencias del Stijl holandés y el & Psicología estructural, epistemología \\
cubismo. & genética, etc. \\
Pas tendencias constructivistas rusas. & Psicología constructivista inglesa. \\
Tendencias racionalistas con origen & constructivista. \\
vernacular (Bruno Taut). Tipismo. & Psicología social interaccionista. \\
Tendencias naturalistas, organicistas y $y$ & (teñida de terminación social). \\
morfobiológicas & Conductismo con base fisiológica. \\
\hline
\end{tabular}

Nota: De la tabla se evidencia una profunda relación entre arquitectura y psicología. Tomado de Psicología y Arquitectura: Notas Breves (Montoñola., 1986, p.37).

Como se puede notar en la Tabla 1, se hace un breve análisis de tendencias arquitectónicas que influyeron en el siglo XX, analizadas y puntualizadas por la objetividad de la psicología donde dignifica las percepciones y el comportamiento del ser humano con su entorno ambiental y social; de allí que la realidad se ha bifurcado, colocando un caso particular ya no se puede denominar familia como un solo tipo, en este momento se cuenta con alrededor de diez tipos de familia, entre las cuales tenemos: familia nuclear, familia monoparental, familia adoptiva, familia sin hijos, familia de padres separados, familia compuesta, familia homoparental y familia extensa; de todas ellas se ha concebido un solo tipo de estructura arquitectónica 
para la formalización de la vivienda, de las otras nueve aún son muy incipientes los desarrollos, más bien son adaptaciones que se ejecutan sobre el modelo de vivienda para familia nuclear.

Es por ello que se requiere de una alianza estratégica entre disciplinas, no tanto para estructurar edificaciones en altura por el supuesto incremento valor de la tierra, sino por el contrario a verificar estructuras de menor altura mucho más aceptables a la realidad familiar y humana, unida con sus necesidades, para que la armonía del hecho arquitectónico sea comunicable, no sea que por el mismo hecho de la falta de interacción social física la realidad edificatoria se vuelva abstracta, en palabras de Montañola "el objeto se vuelve "incomunicable" en la medida que el sujeto se vuelve "interiorizado" " (1986, p.38).

Por otra parte, la historia de la arquitectura no se ha detenido y las tendencias arquitectónicas nuevas y novedosas existen, falta actualizarlas en incluso recibir un acompañamiento por parte de las Ciencias Sociales para interpretarlas y reconocer su gran funcionalidad y compromiso para con el ser y su entorno.

\section{CONCLUSIONES}

Al responder la pregunta: ¿Arquitectura, parte integral de las ciencias sociales?, se ha realizado un recorrido por dos aspectos fundamentales: Arquitectura más allá de la espacialidad, donde se realizó un recorrido por diferentes tesis donde tratan de concretar el que es ser arquitecto y, que es arquitectura, donde posiblemente se busca un nuevo acercamiento al definir arquitectura de forma más holística: "Es aquella área del conocimiento que, de manera formal, recrea el espacio adaptándolo a las necesidades humanas, sentidas del lugar, y cuya interpretación del hecho u objeto arquitectónico se crea a través de nueve lenguajes manifestándose en una formalidad espacial, interrelacionada con el usuario en su estado de permanente o transeúnte; esta arquitectura se apoya de las habilidades de ingeniería en términos edificatorios y en las ciencias sociales para el conocimiento del ser en su medio físico y social”.

Es por eso, que las Ciencias Sociales, son tan importantes a la arquitectura y no se deben desligar ya que la originalidad en el proyecto arquitectónico, se presenta de un buen análisis de la población a la cual se dirige pues en cada caso y en cada lugar los valores humanos cambian, generando con ello una riqueza compositiva que enmarca la tendencia de la nueva realidad social, que posiblemente nos dio como punto de partida este aislamiento repentino causado por una pandemia, sin embargo, se ha podido evidenciar que la raza humana es social por excelencia y que por más individualismo existente en algunas labores, esta se complementan en la interactuación entre seres de forma más presencial.

Se deja como inicio de partida al debate, hasta qué punto el arquitecto debe profundizar sobre el conocimiento de las Ciencias Sociales, o si más bien se debe buscar la transversalidad entre las disciplinas apoyándose y compartiendo conocimientos para que la arquitectura sea más provechosa entre áreas buscando una sostenibilidad no solamente ambiental, sino social y educacional.

\section{REFERENCIAS}

Benévolo, L. (1982.). Historia de la Arquitectura Moderna. Barcelona.: Gustavo Gili.

Jiménez, F \& Aragonés, J. (1986). Psicología y Arquitectura. Notas Breves. En Introducción a la Psicología Ambiental. (pp 34-41.). Madrid.: Alianza Editorial, S.A.

Jackson, W. (1953.). Enciclopedia Práctica Jackson. Buenos Aires.: Impresores, The Colonial Press Inc.

Montoñola, J. (1986). Psicología y Arquitectura. Notas Breves. Capitulo 2. Alianza Editorial, S.A.

Patteta, L. (1997.). Historia de la Arquitectura (Antología Crítica). Madrid.: Celeste Ediciones. 
Ley 435/98, febrero 19, 1998. Diario Oficial. [D.O.]: 43.241. (Colombia).

\section{SEMBLANZA DE LOS AUTORES}

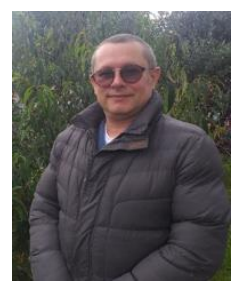

Fabian E. Báez Álvarez: Obtuvo el grado de Arquitecto en la Universidad La Gran Colombia, Colombia, donde actualmente es profesor vinculado. Desarrolló sus estudios de Postgrado en Derecho Administrativo y Maestría en Educación con Énfasis en Ciencias Sociales, en la Universidad Externado de Colombia. Ha desarrollado proyectos arquitectónicos en la ciudad de Villavicencio Meta, su vinculación con la docencia la ha desarrollado desde hace más de veinte años, ha participado en varios proyectos de investigación, además forma parte de la Fundación Educativa y Social JAP y de la Unión de Escuelas y Facultades de Arquitectura de América Latina - UDEFAL.

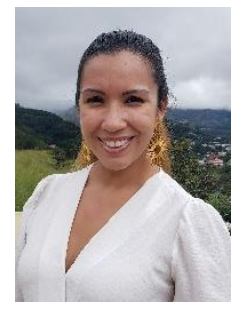

Katherine Del C. González Rivera: Obtuvo el grado de Arquitecta Estructural en la Universidad Santa María La Antigua, Panamá. Actualmente es profesora en la Universidad Autónoma de Chiriquí. Desarrolló sus estudios de Postgrado en Docencia Superior en Columbus University, Panamá; Maestría en Administración de Proyectos de Construcción en la Universidad Santa María la Antigua; y Doctorado en Arquitectura en American Andragogy University, Estados Unidos. Ha desarrollado proyectos arquitectónicos en la ciudad de David, Chiriquí principalmente; su vinculación con la docencia la ha desarrollado desde hace más de doce años, forma parte de la Asociación de Arquitectos de Chiriquí, y de la Unión de Escuelas y Facultades de Arquitectura de América Latina - UDEFAL. 